\title{
TM 2: Assembly prototype girder
}

\author{
S. Brooks, S. Peggs, D. Trbojevic, J. Tuozzolo
}

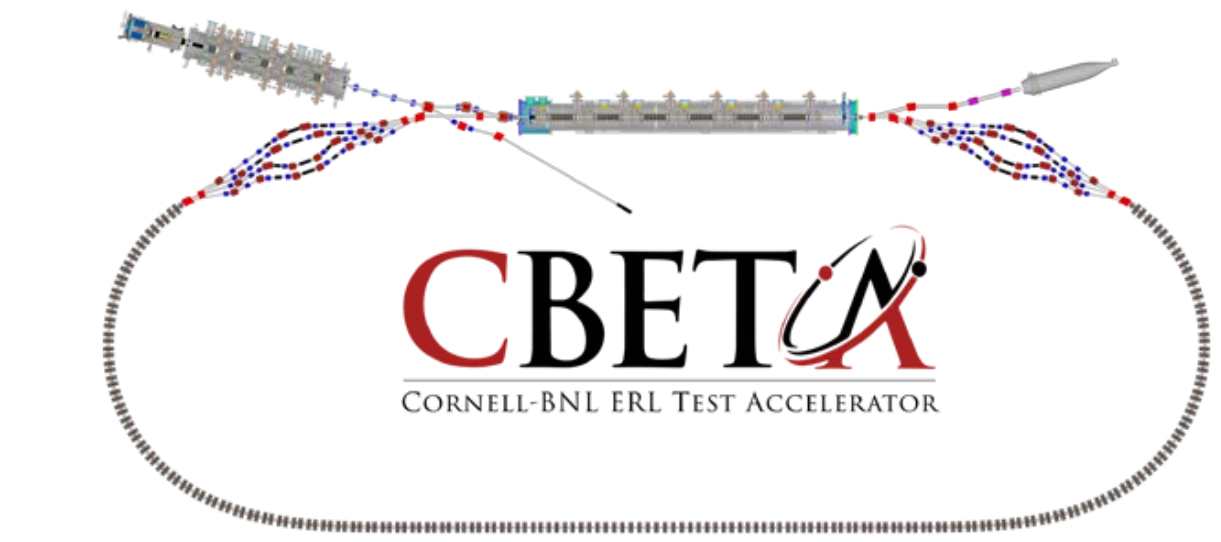

Collider-Accelerator Department, Brookhaven National Laboratory, Upton NY 11973 U.S. Department of Energy Office of Science, Office of Nuclear Physics

Cornell Laboratory for Accelerator-Based Sciences and Education, Ithaca, NY 14850

Funded by NYSERDA contract 102192

Notice: This document has been authorized by employees of Brookhaven Science Associates, LLC under Contract No. DE-SC0012704 with the U.S. Department of Energy. The United States Government retains a non-exclusive, paid-up, irrevocable, world-wide license to publish or reproduce the published form of this document, or allow others to do so, for United States Government purposes. 


\section{DISCLAIMER}

This report was prepared as an account of work sponsored by an agency of the United States Government. Neither the United States Government nor any agency thereof, nor any of their employees, nor any of their contractors, subcontractors, or their employees, makes any warranty, express or implied, or assumes any legal liability or responsibility for the accuracy, completeness, or any third party's use or the results of such use of any information, apparatus, product, or process disclosed, or represents that its use would not infringe privately owned rights. Reference herein to any specific commercial product, process, or service by trade name, trademark, manufacturer, or otherwise, does not necessarily constitute or imply its endorsement, recommendation, or favoring by the United States Government or any agency thereof or its contractors or subcontractors. The views and opinions of authors expressed herein do not necessarily state or reflect those of the United States Government or any agency thereof. 


\section{TM 2: Assemble Prototype Girder}

\section{Contents}

1 Acknowledgments $r$

2 Introduction $\quad 2$

3 Mechanical analysis, forces, design and alignment $r$

4 Magnetic testing and performance $\quad 5$

5 Thermal variation and stability $r$

\section{Summary}

This Technical Note presents details of the successful achievement of Technical Milestone 2 in the NYSERDA contract with Brookhaven National Laboratory and Cornell University. The project - to design, build and commission the Cornell-BNL Electron Test Accelerator (CBETA) - will achieve final success when all 12 Technical Milestones are complete. Milestone 2 marks the completion of Task 2, defined as:

\section{Task 2: Assemble Prototype Girder}

Task Goal: Assemble and test the prototype girder using 8 hybrid magnets.

Deliverables:

- An assembled prototype girder to be used in the accelerator circumference made of 8 hybrid magnets blocks embedded into iron poles and back-legs.

- Perform rigorous magnetic, mechanical, alignment and thermal tests on the prototype and document the results of the tests.

The assembly and testing of the prototype girder and its components are documented below.

\section{Acknowledgments}

The authors of this note are just the individuals who participated directly in writing and editing. We are glad to acknowledge the hard work and support of many other people who contributed to the successful assembly of the prototype girder, including Henry Ashby, Steven Bellavia, Scott Berg, Cyrus Biscardi, John Cintorino, Michael Hartsough, Robert Karl, Robert Lehn, George Mahler, Francois Meot, Gary McIntyre, Rob Michnoff, Humberto Palacios, Thomas Roser, John Siano, Charkes Trabocchi, Steven Trabocchi, and Nick Tsoupas. 


\section{Introduction}

Figure 1 shows the fully assembled girder, including eight magnets surrounding an integrated vacuum beampipe. The integrated assembly rests on a specialized table - a girder. Each magnet consists of a Halbach core surrounded by a relatively weak electromagnet corrector. The magnetic field in the Halbach magnets is driven by a set of many permanent magnet blocks that are assembled in a complete circular arc surrounding the beampipe, as discussed in Section 3.

The "Task Goal" explicitly states that 8 hybrid magnets will be assembled on the girder. The implicit goal is to include 8 permanent magnets (not electromagnets) in a configuration that provides two Fixed Fileld Alternating Gradient (FFAG) optical cells, following the same layout that will be used in the CBETA return arcs. Two technological choices are available for these magnets: "hybrid" or "Halbach". In January 2017 it was decided to switch from hybrid to Halbach technology. The intent of the Task Goal has been met using Halbach magnets, not hybrid magnets.

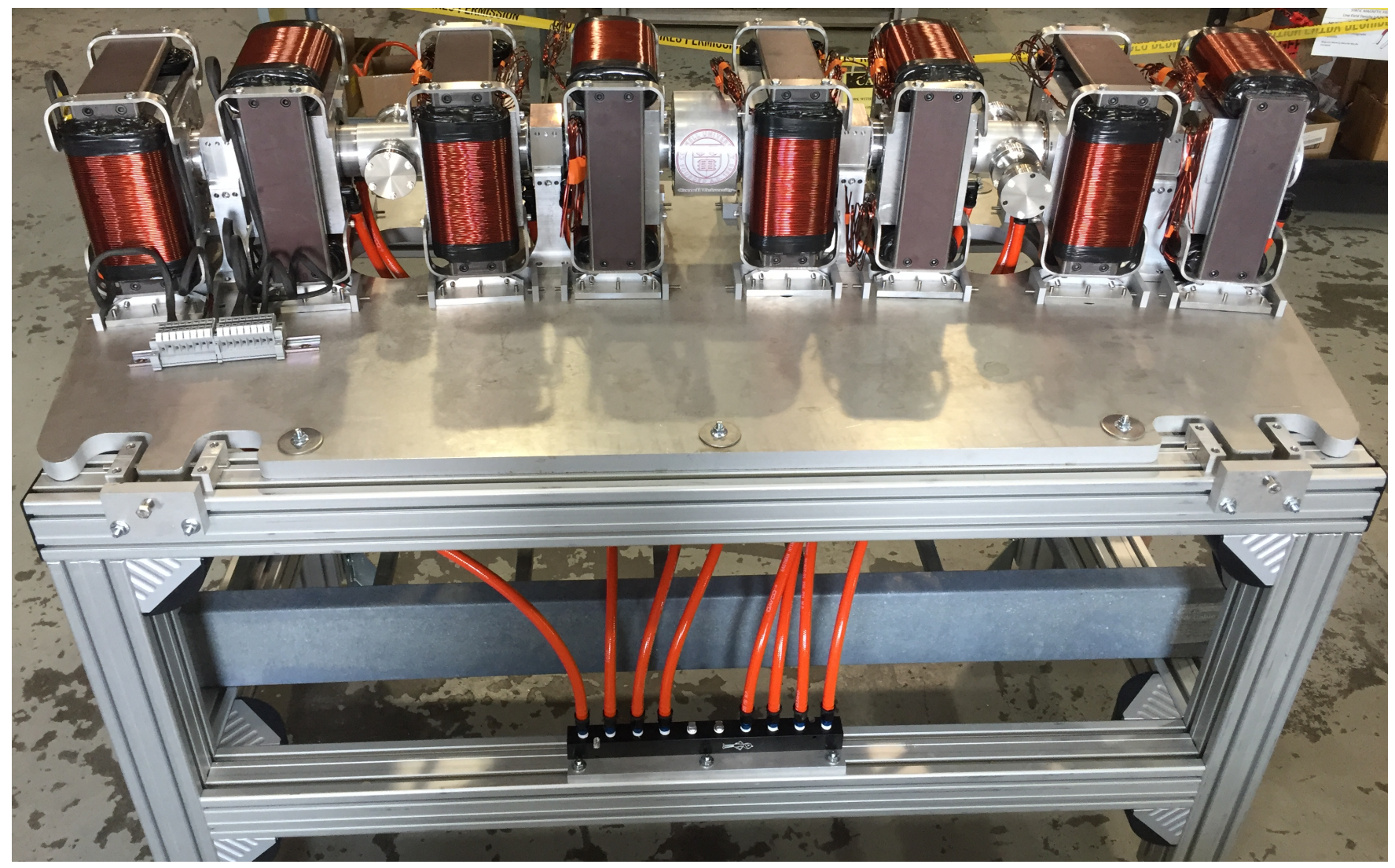

Figure 1: The prototype girder shown in this figure supports eight Halbach magnets, each surrounded by electromagnetic correctors, constructed around a vacuum beampipe. The fully integrated assembly of many components is carefully aligned on the girder.

\section{Mechanical analysis, forces, design and alignment}

This pre-production "first" girder supports two types of Halbach magnet: a circularly symmetric quadrupole "QF" and a lopsided combined quadrupole plus dipole "BD". There are four magnets of each type on the girder.

Halbach proof-of-principle magnets were constructed and tested to verify the ability of these magnet types to meet the CBETA technical and installation requirements as well as the magnetic field quality requirements discussed in Section 4. Another goal was to design and build a double cell assembly (8 magnets, 4 magnets/cell) that would 


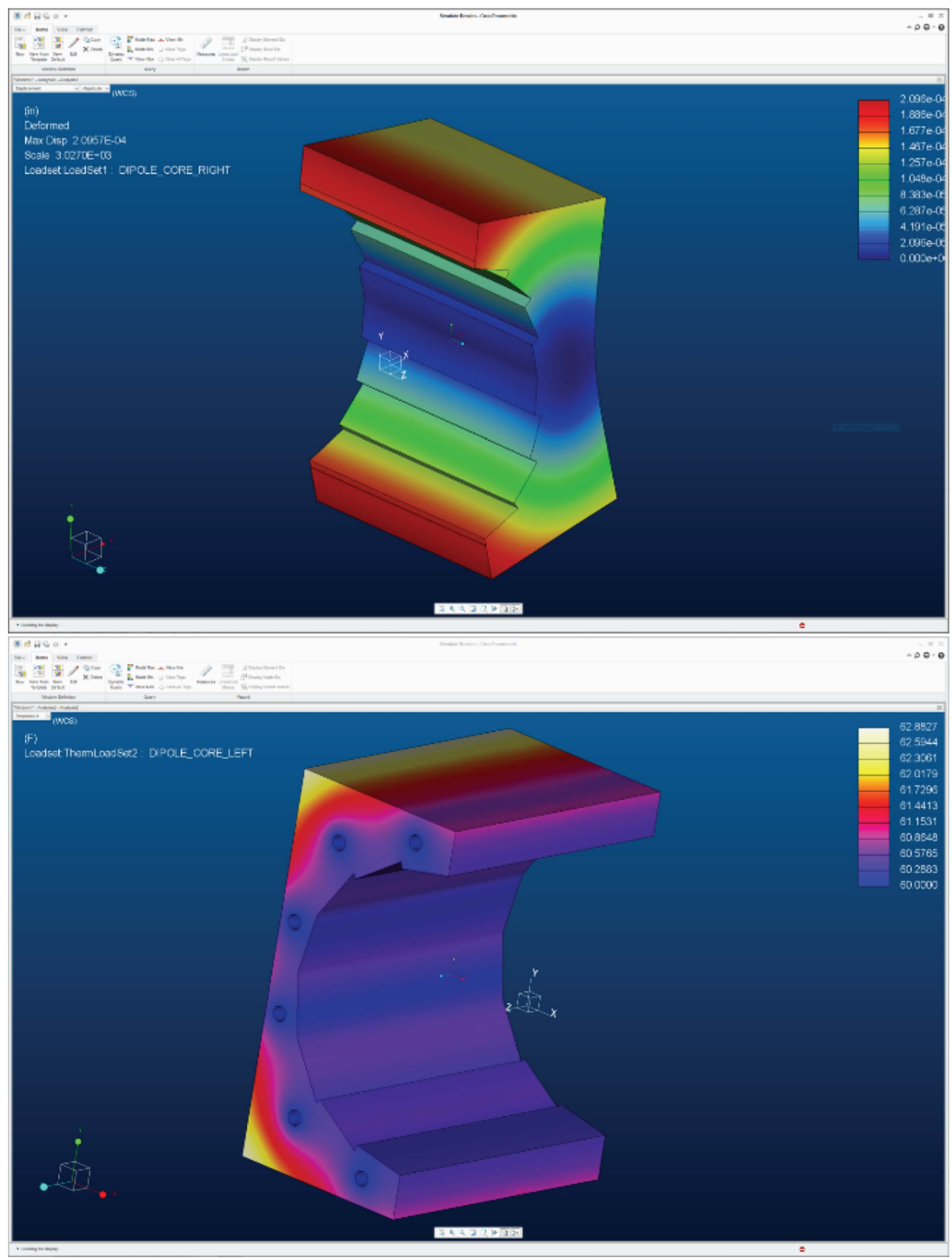

Figure 2: Mechanical analysis of the deflection of an aluminum frame of a QF magnet (top) and the temperature distribution with water passages (bottom).

match the requirements for a production cell assembly to be installed in CBETA, for the fractional Arc Test in early 2018.

The pre-production assembly incorporated features that will be used on the production magnets to meet these goals:

1. The magnets were built with aluminum outer frames that are split on the vertical plane, for ease of vacuum chamber installation and maintenance. The split allows installation of a complete and tested vacuum chamber assembly with beam position monitors pre-installed and tested. The aluminum frames provide high strength and low deflection when resisting the magnetic forces from the permanent magnet material (PMM) blocks. The frames were machined with tight tolerances to enable accurate block positioning.

2. The aluminum frames were cross drilled with water passages for temperature stabilization of the PMM blocks. Aluminum provides excellent thermal conductivity enabling small temperature variations around the outer circumference of the PMM ring.

3. The magnet assemblies were designed to be built with brass shims that were used to vary the inner radius of 


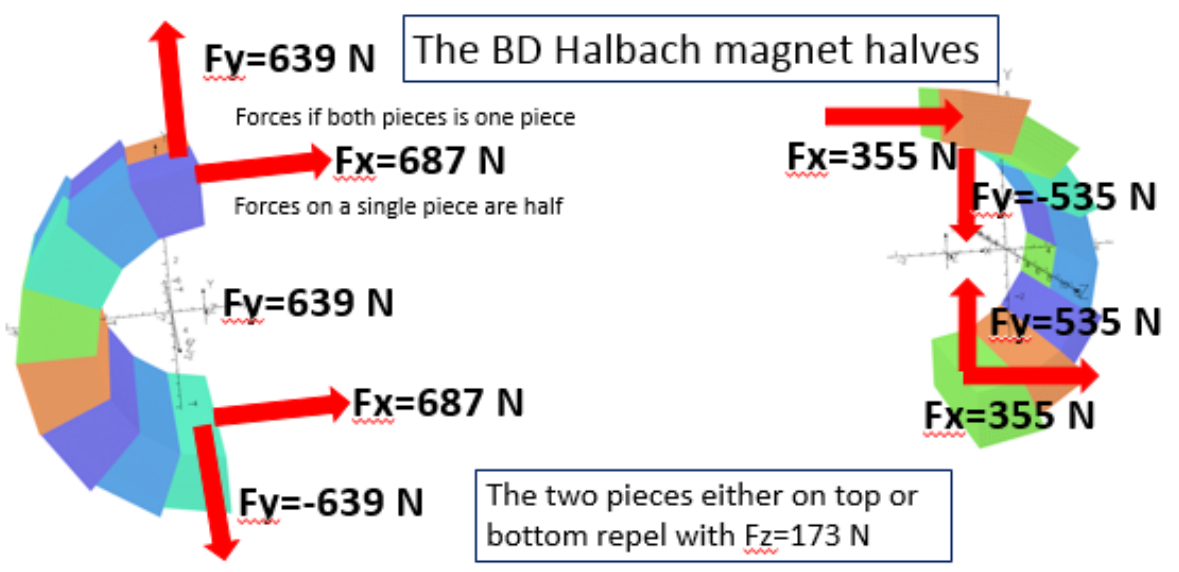

Figure 3: Forces between PMM blocks, on the aluminum frame and on the epoxy, for the most challenging BD Halbach magnet style.

the PMM ring, and therefore the strength of the magnetic field at the center of the beam pipe, to deal with the possible block-to-block variations of the PMM field strengths.

4. The Halbach magnets use epoxy to hold the PMM blocks in place, for example when the magnets are split for installation or maintenance. Forces on the individual blocks can be as high as $150 \mathrm{lbf}$, and the stress on the epoxy can be as high as 100 psi.

Prior to manufacturing the aluminum frames, they were analyzed with the ANSYS finite element thermal and stress analysis program. Their design was modified and optimized to reduce deflection from the magnetic forces and provide an even temperature distribution. Figure 2 shows samples of the analyses performed for deflection and temperature distribution. All three magnet frame types were analyzed for stress, deflection, and temperature stability.

The block-to-block forces between PMM blocks result in forces on the aluminum frame, and on the epoxy that is tasked with holding the blocks to each other and to the aluminum frame. The analysis of these forces was used for the QF deflection analysis shown in Figure 2, and to verify that the stress on the epoxy was within its design values. Figure 3 shows the analysis for the BD Halbach magnet style - the style that has the highest force on individual end

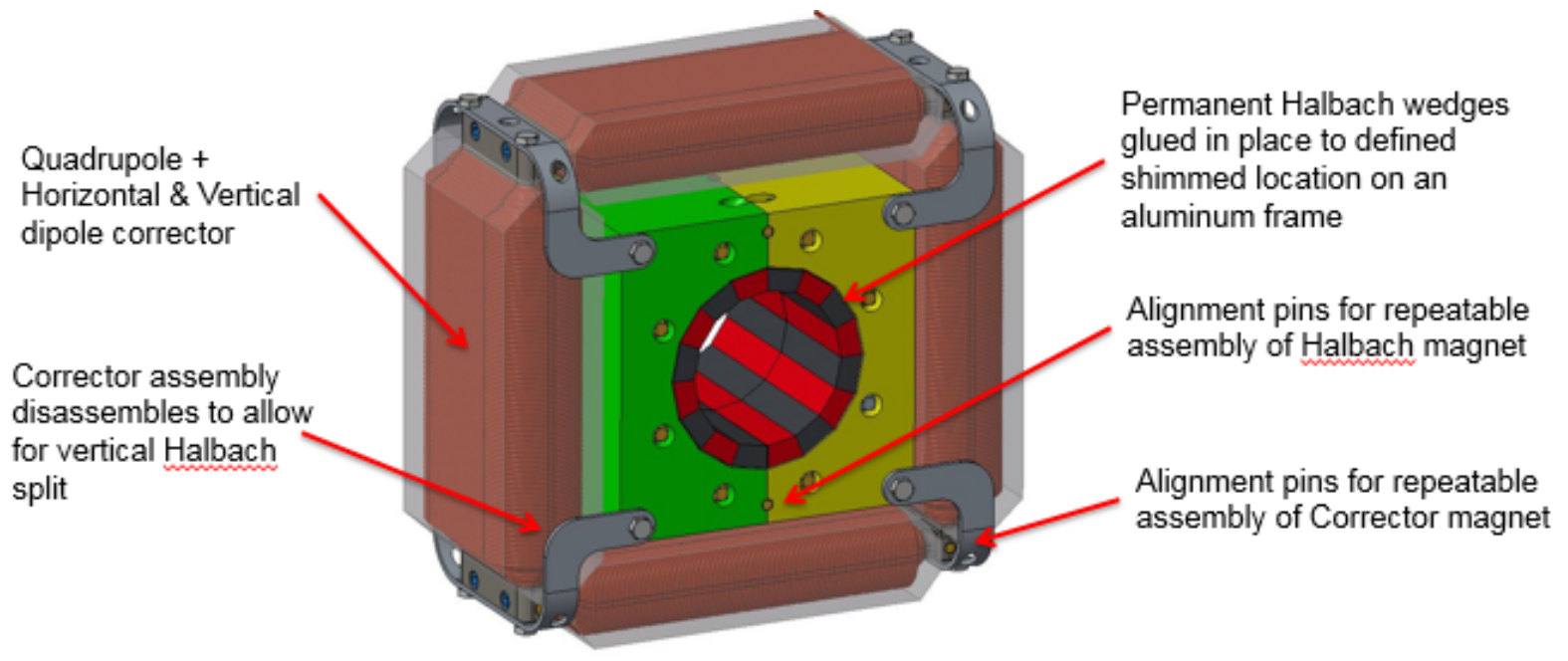

Figure 4: Design model showing the assembly brackets and alignment dowels (pins) used during magnet assembly. 
blocks: $343 \mathrm{~N}$ (77 lbf) per block. With a block surface area of 2.2 square inches, the tensile stress on the epoxy is less than 100 psi. Tensile and shear bond strengths for epoxy typically exceed 1000 psi. Properly prepared surfaces and cured epoxy worked well on the pre-production magnets. Adhesion tests will be a requirement for the production magnets.

The magnet frames were manufactured with alignment dowels that ensured repeatability throughout the measurement process, despite separating and reuniting the two halves multiple times. The measurement results were consistent throughout the measurement the tuning process. Figure 4 shows the design model, including the assembly brackets and alignment dowels used during magnet assembly.

The design of the girder that supports the 8 FFAG magnets was significantly simplified when the decision was made to convert from hybrid (steel/PMM) technology to Halbach technology, because the Halbach magnets are 1/10 of the weight. The magnets for the preproduction magnet assembly have been mounted on an aluminum plate that was accurately machined using a CNC water jet cutter, including access holes to allow water lines to enter, and to aid in passively cooling the correctors. Taped holes and smaller mounting holes can be located with a single CNC operation for the magnet mounting points.

Accurately machined Halbach magnet frames enable accurate PMM block positioning, and accurate assembly of the two magnet halves. Accurate alignment of the integrated magnet assemby is achieved with multi-position jacking and alignment screws that can be locked into place after survey. The model and photo shown in Figure 5 illustrate the kinematic mount for the magnets. A lesson learned during construction was to modify the bracket to provide more space for the vertical corrector coil.
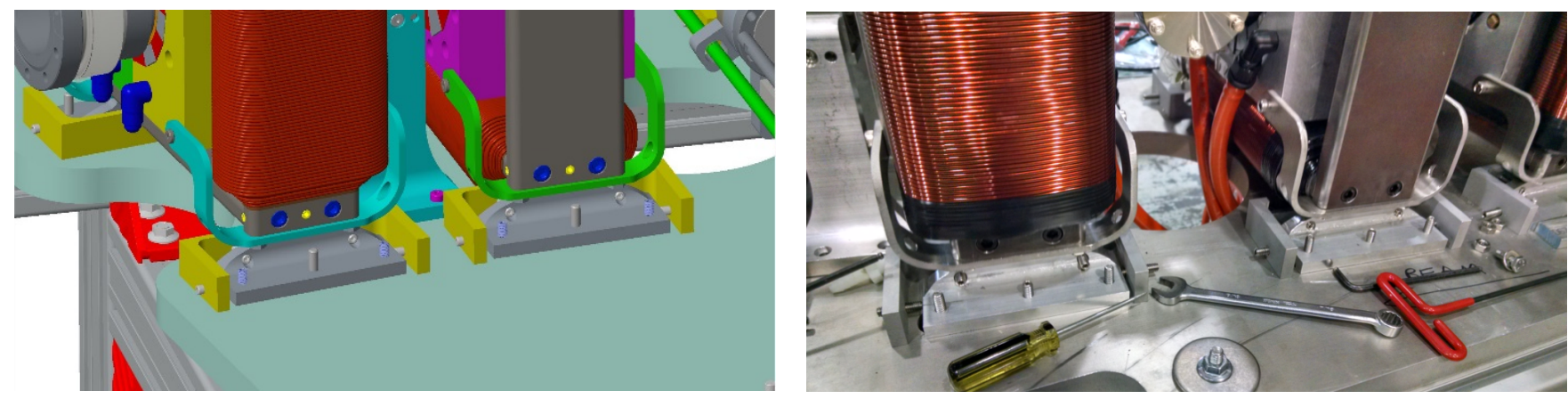

Figure 5: The kinematic mount at the base of the integrated magnet assembly enables accurate alignment.

\section{Magnetic testing and performance}

Magnetic measurements were made at two stages in production, in order to trace potential errors during the assembly of the first girder:

1. On individual permanent magnet blocks. Each block has a different magnetization strength and direction.

2. Across the aperture of assembled magnets, each containing 32 blocks.

Individual permanent magnet blocks were measured using a Helmholtz coil set-up that gave a 3-dimensional vector of the direction and strength of magnetization. Figures 6 and 7 show the distributions of magnetization angle and strength, obtained from all 384 permanent magnet blocks used in the first girder.

Block magnetization angle errors are particularly important. The root mean square error of the full distribution shown in Figure 6 is less than one degree, as desired. The two families of BD magnet blocks labeled "BD small pieces" and "BD smallest pieces" have larger systematic errors than the rest, but a have a relatively small contribution to the overall magnetic field, and field quality, due to their small size.

The block magnetization strengths shown in Figure 7 have a standard deviation of $0.429 \%$ for the QF blocks, and a standard deviation of $0.466 \%$ for the "BD large pieces" blocks, both better than the desired value of $0.6 \%$. The average value of $1.182 \mathrm{~T}$ is within the range of $1.17-1.22 \mathrm{~T}$ that is expected by the manufacturer. This average strength was used to determine the thicknesses of the brass shims that were inserted around the magnet blocks to finely adjust the strength of the assembled magnet. 


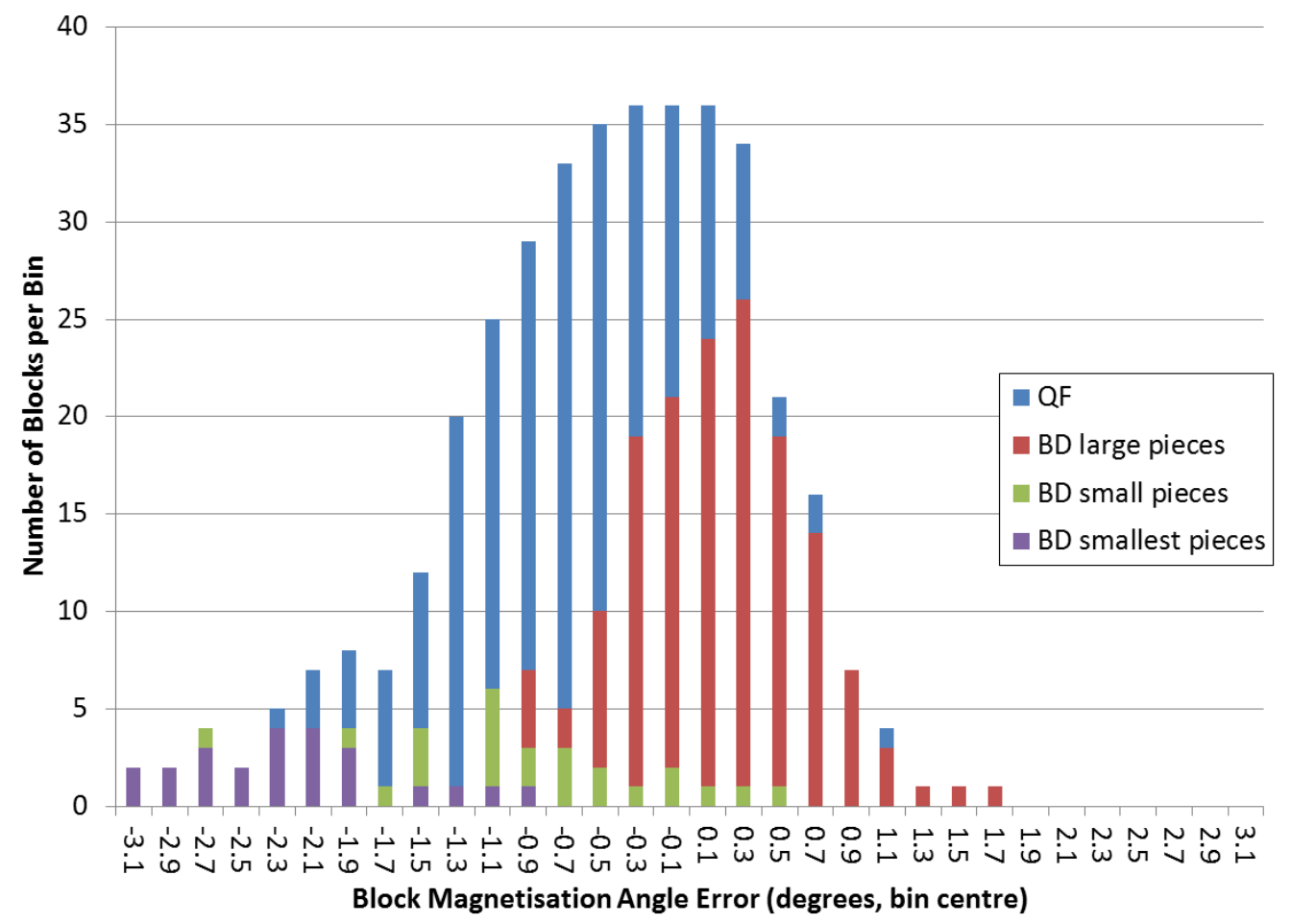

Figure 6: Magnetization angle errors measured by Helmholtz coil across all permanent magnet blocks.

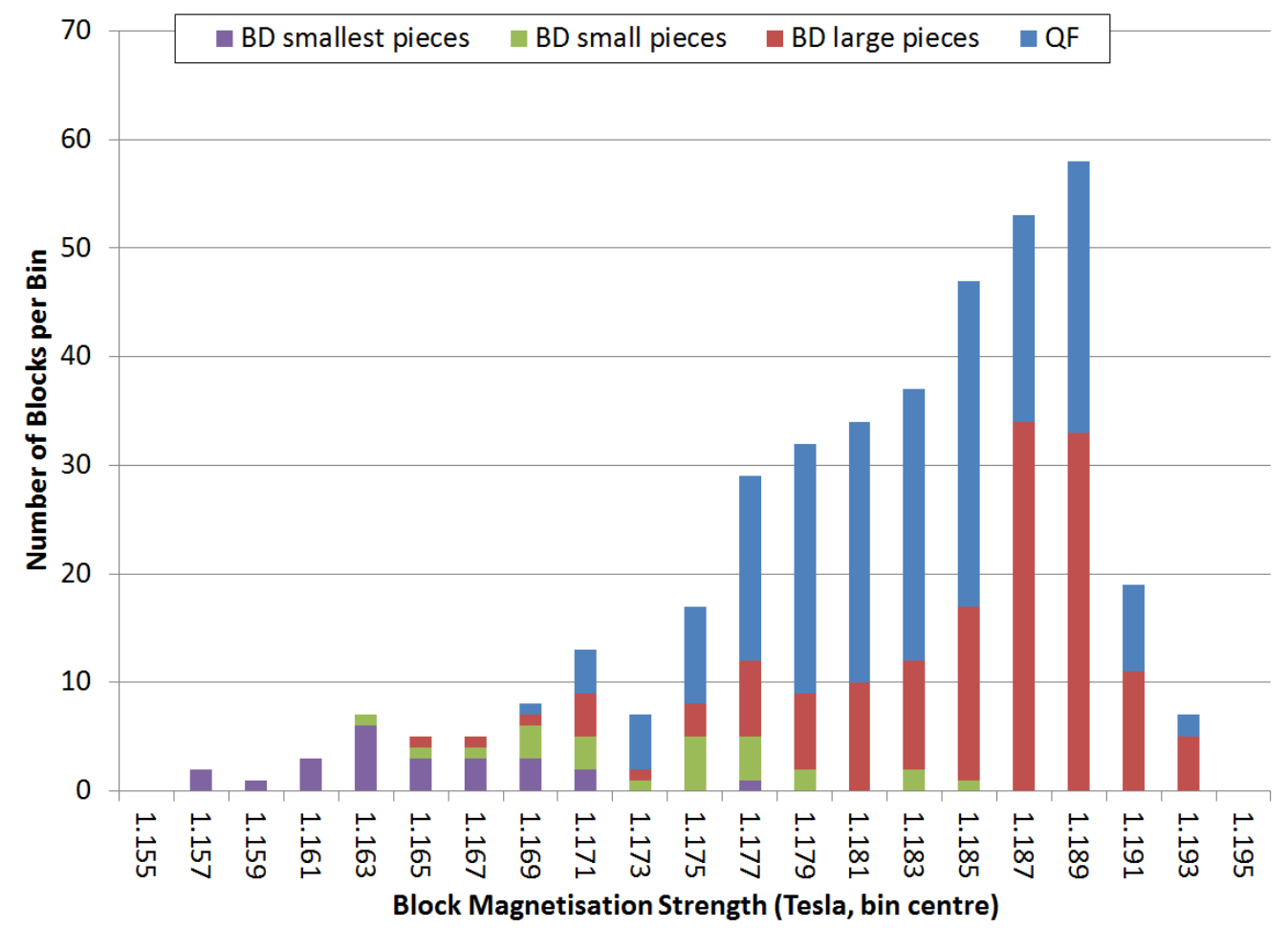

Figure 7: Distribution of magnetization strength measured by Helmholtz coil across all permanent magnet blocks. 


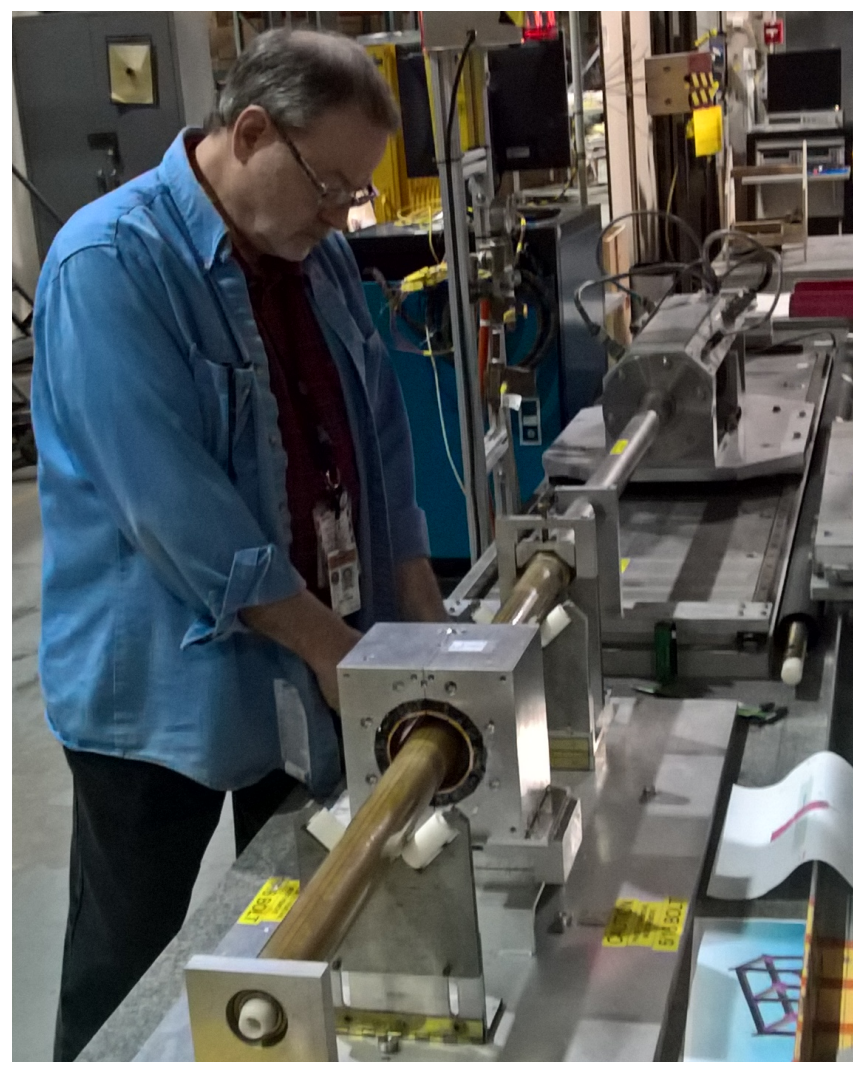

Figure 8: John Cintorino operates a rotating coil at BNL to measure the multipoles of a QF magnet (bottom center).

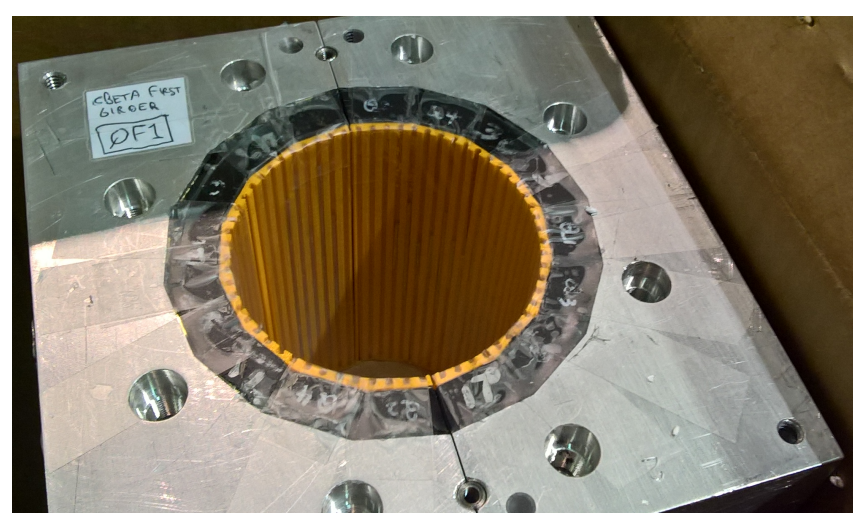

Figure 9: Iron tuning wires of adjustable length were placed inside the slots in the orange plastic 3D printed cartridge that is visible in this photograph, inside the bore of the QF1 magnet, in order to cancel out field imperfections.

The second set of magnetic measurements - across the aperture of each individual magnet - was performed on fully-assembled magnets, using a rotating coil, as illustrated in Figure 8. The rotating coil goes through the magnet bore, measuring the field quality as a set of "multipoles". The multipoles of each magnet were measured after the two halves were assembled for the first time. In order to reduce the field imperfections to a level acceptable for accelerator-quality magnets - less than $0.1 \%$ error per multipole - the magnets were then retuned, as shown in Figure 9. A set of iron wires of adjustable length was inserted into slots in the orange plastic cartridge inside the magnet bore. Figure 10 shows a multipole reduction by about a factor of 20 after retuning, to a level that is about a factor of 4 better than required. 


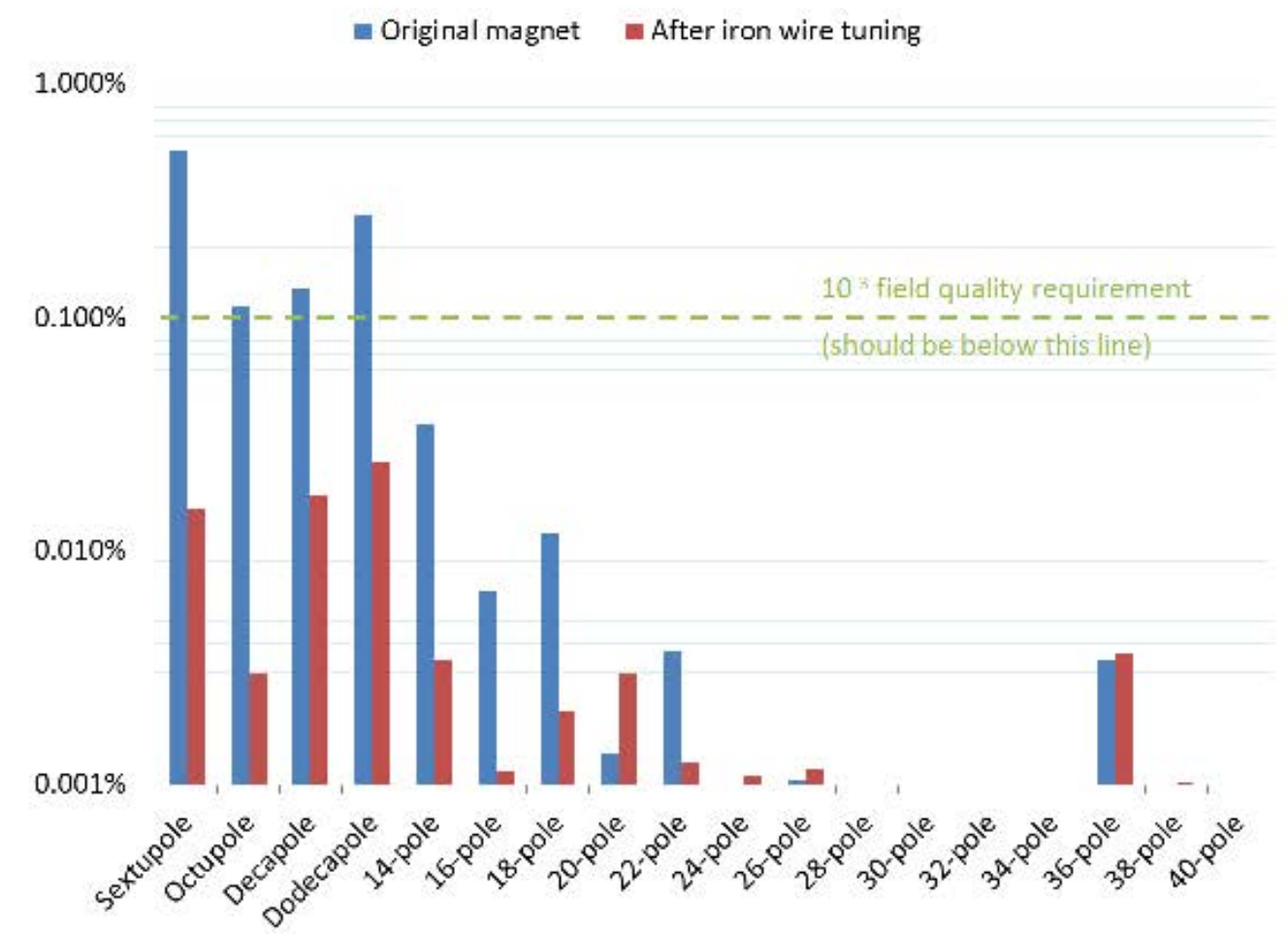

Figure 10: Multipole field errors measured in magnet QF1, before and after tuning with adjustable length iron wires.

\section{Thermal variation and stability}

The CBETA magnets will be operated at a controlled temperature because the neodymium-iron-boron ( $\mathrm{NdFeB})$ permanent magnet material has a magnetization strength that varies with temperature. The thermal coefficient of this material is $-0.11 \%$ per degree Kelvin temperature rise. To account for this, the temperature of the magnet was recorded each time the rotating coil field measurement was performed. The results are shown in Figure 11, which plots magnet strength against temperature.

The field strengths of the magnets must be extrapolated from the measurement temperature to the CBETA running temperature of $85^{\circ} \mathrm{F}$, the temperature of the cooling water circuit. The field strengths are significantly closer to the target strength once this correction is performed, with strength differences at a level - less than $1 \%-$ that is correctable by other means, either by wire tuning or by installing quadrupole correctors. 


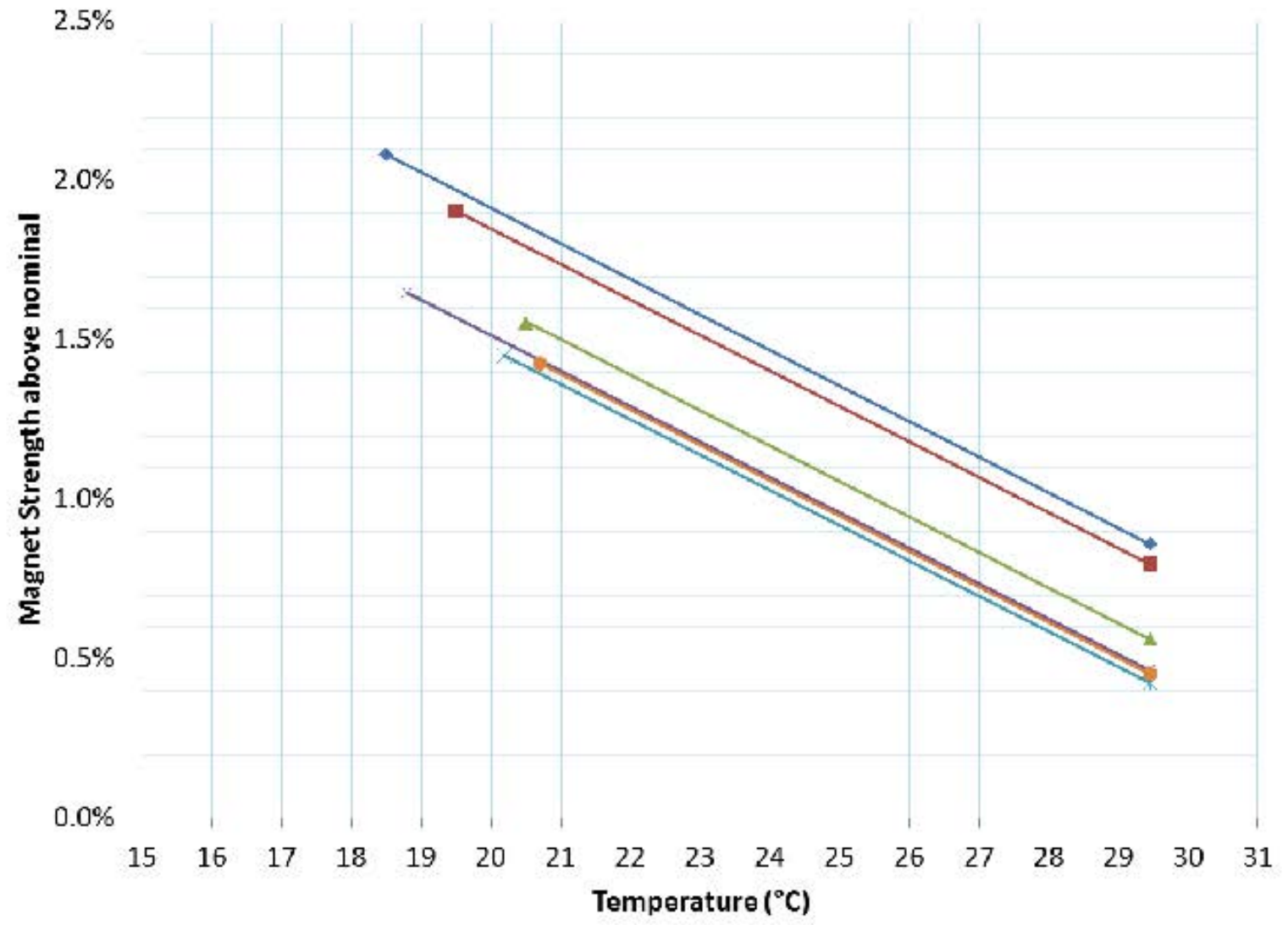

Figure 11: Thermal behaviour of magnet strengths, extrapolating from measurement temperatures in the range $18.5^{-}-20.6^{\circ} \mathrm{C}$ to the operating temperature of $29.4^{\circ} \mathrm{C}\left(85^{\circ} \mathrm{F}\right)$. 